\title{
The Degrees of Freedom of Full-Duplex Bi-directional Interference Networks with and without a MIMO Relay
}

\author{
Zhiyu Cheng, Natasha Devroye, Tang Liu \\ University of Illinois at Chicago \\ zcheng3, devroye, tliu44@uic.edu
}

\begin{abstract}
In a full-duplex bi-directional interference network with $2 K$ transceivers, there are $K$ communication pairs: each user transmits a message to and receives a message from one intended user and interferes with and experiences interference from all other users. All nodes may interact, or adapt inputs to past received signals, and may thus cooperate with each other. We derive a new outer bound, and use interference alignment to demonstrate that the optimal degrees of freedom (DoF, also known as the multiplexing gain) is $K$ : full-duplex operation doubles the DoF, but interaction and cooperation does not further increase the DoF. We next characterize the DoF of a full-duplex bi-directional interference network with a MIMO, full-duplex relay. If the relay is non-causal/instantaneous (at time $k$ forwards a function of its received signals up to time $k$ ) and has $2 K$ antennas, we demonstrate a one-shot scheme where the relay mitigates all interference to achieve the interference-free $2 K \mathrm{DoF}$. In contrast, if the relay is causal (at time $k$ forwards a function of its received signals up to time $k-1$ ), we show that a fullduplex MIMO relay cannot increase the DoF of the full-duplex bi-directional interference network beyond $K$, as if no relay or interaction is present.
\end{abstract}

\section{INTRODUCTION}

In wireless communications, current two-way systems often employ either time or frequency division to achieve two-way or bidirectional communication. This restriction is due to a combination of hardware and implementation imperfections and effectively orthogonalizes the two directions, rendering the bidirectional channel equivalent to two one-way communication systems. However, earlier work [3], [4] as well as recently much progress has been made on the design of fullduplex wireless systems [5]-[8], which show great promise for increasing data rates in future wireless technologies. The next generation mobile networks (NGMN) Alliance has defined $5 \mathrm{G}$ network requirements which include data rates of several tens of $\mathrm{Mb} / \mathrm{s}$ for tens of thousands of users, which might be achieved through densification of networks and the usage of massive amounts of antennas, among other technologies. In this work we seek to understand the potential of full duplex systems, with and without multi-antennas relays, in wireless environments with possibly significant amounts of interference (motivated by for example extreme densification of networks).

This paper was presented in part at [1] and [2]. The work of N. Devroye and Z. Cheng was partially supported by NSF under awards 1216825 and 1053933. The contents of this article are solely the responsibility of the authors and do not necessarily represent the official views of the NSF.
We do so from a multi-user information theoretic perspective by obtaining the degrees of freedom of several full-duplex bidirectional networks with and without relays.

Full-duplex operation enables true two-way communications over the practically relevant Gaussian noise channels. We currently understand the theoretical limits of a point-topoint, full-duplex Gaussian two-way channel where two users wish to exchange messages over in-band Gaussian channels in each direction: the capacity region is equal to two independent Gaussian noise channels operating in parallel [9]. Full duplex operation thus roughly doubles the capacity of this simple twoway network.

To extend our understanding of the impact of full-duplex operation to two-way networks with interference, the two-way interference channel (TWIC) has been studied in [10]-[13], in which there are 4 independent messages: two-messages to be transmitted over an interference channel (IC) in the $\rightarrow$ direction simultaneously with two-messages to be transmitted over an in-band IC in the $\leftarrow$ direction. In wireless communications, the TWIC has been used in [14] to model an inter-cell interference scenario where a base station in one cell suffers interference signal from a user in an adjacent cell and vice versa. In our information theoretic model, all 4 nodes in the network act as both sources and destinations of messages. This allows for interaction between the nodes: a node's channel inputs may be functions of its message and previously received signals. The capacity region of the point-to-point two-way channel is still open in general, though we know the capacity for the Gaussian channel. Similarly, the capacity region of the one-way IC is still open, though we know its capacity to within a constant gap for the Gaussian noise channel [15]. In general then, finding the full capacity region of the full-duplex TWIC is a difficult task, though progress has been made for several classes of deterministic channel models [11], and capacity is known to within a constant gap in certain parameter regimes and adaptation constraints [11], [12], [16].

The degrees of freedom (DoF) [17] of a network provide an approximate capacity characterization that intuitively corresponds to the number of independent interference-free signals that can be communicated at high signal to noise ratios (SNR). The DoF have been of significant recent interest in one-way interference networks [18], [19]. Here, we seek to extend our understanding of the DoF to two-way networks, whose study is motivated by the fact that full-duplex operation is 
becoming practically realizable. Some progress has already been made: the DoF of the full-duplex TWIC has been shown to be 2 [10], [12]. This is interesting, because the capacity of any network with interaction at nodes is no smaller than that of the same network where interaction is not possible (interaction can mimic non-interaction). However, that the TWIC with interaction has only 2 DoF demonstrates that interaction between users does not increase the DoF of the two-way IC beyond the doubling that full-duplex operation provides. We ask whether the same is true for the more general, full-duplex bi-directional interference network with and without a MIMO relay node.

\section{A. Contributions}

In this work, we first propose and study a natural extension and generalization of the (2-pair-user) two-way interference channel (TWIC): the ( $K$-pair-user) full-duplex interference network, i.e., there are $2 K$ messages and $2 K$ users forming a $K$-user IC ( $K$ messages) in the forward direction and another $K$-user IC in the backward direction ( $K$ messages). All $2 K$ users interfere with, or alternatively may cooperate with, one another. We consider this $2 K$ node network with and without the presence of a MIMO relay. All nodes may employ interaction - i.e. signals may be a function of previously received outputs. Compared to the 2-pair-user IC, the nodes in the $K$-pair-user full-duplex bi-directional interference network experience interference from significantly more users: due to the adaptation and cooperation involved, all users see a combination of the signals of all other $2 K-1$ users in addition to seeing self-interference (SI) signals, which are transmitted by the user itself or received via other signals due to adaptation. Canceling SI is one of the main challenges in real full-duplex wireless systems. However, in this theoretical work for the Gaussian channels involved, the self-interference is known to the receiver and as such, theoretically, it can be subtracted off. We then explore the limits of communication under the assumption that this self-interference may be removed. Our main results are:

1) We first show that the sum degrees of freedom of the $K$ pair-user full-duplex bi-directional interference network is $K$, i.e. $K / 2$ in each direction, for both time-varying and (almost all) constant channel coefficients. In other words, each user still gets half a DoF and neither interaction nor cooperation between users - even though our outer bound permits it increases the sum DoF. Intuitively this is because all the links in the network have similar strengths in the DoF sense, so that a user cannot "route" other users' desired signals through cross or direct links since they are occupied by its own data signals. In addition, coherent power gains which may be the result of adaptation and the ability of nodes to correlate their channel inputs, do not affect the DoF (i.e. coherent power gains for Gaussian channels lead to additive power gains inside the logarithm rather pre-log, or DoF/multiplexing gains). The contribution lies in the outer bound - which is valid for nodes which may adapt and does not immediately follow from known two-user interference channel results - and applying interference alignment originally derived for the one-way $K$ user IC as a new achievability scheme. Full-duplex operation is thus seen to double the DoF, but interaction and cooperation is not able to increase the DoF beyond this.

2) We next consider the full-duplex bi-directional interference network with an additional, multi-antenna, full-duplex relay node that seeks to aid the communication of the $K$-pair users. We ask whether the presence of such a relay node may increase the DoF. Interestingly, we show that while the DoF of the $K$-pair-user full-duplex bi-directional interference network is $K$ - indicating that interference is present and somewhat limits rates - that the presence of an instantaneous MIMO relay with $2 K$ antennas may increase the DoF to the maximal value of $2 K$, i.e. each user in the network is able to communicate with its desired user in a completely interferencefree (in the DoF sense) environment. The key assumption needed is for the relay to be non-causal or instantaneous meaning that at time $k$ it may forward a signal based on the received up to and including time $k$. We see that full-duplex operation combined with instantaneous / non-causal relaying with multiple antennas may in this case quadruple the DoF over the one-way $K$-user IC.

3) Finally, we show a result which is in sharp contrast to the previous point: if the relay is now causal instead of noncausal, meaning that at time $k$ it may only forward a signal which depends on the received signals up to and including time $k-1$, then we derive a novel outer bound which shows that the DoF of the $K$-pair-user full-duplex bi-directional interference network with a causal MIMO relay is $K$ (regardless of the number of antennas at the relay). This is the same as that achieved without a relay, and without interaction. In summary, full-duplex operation again doubles the DoF, but a causal, full duplex relay is unable to increase the DoF beyond that.

\section{B. Related Work}

The degrees of freedom of a variety of one-way communication networks have been characterized [19]-[23], a complete survey of this large area is beyond the scope of this work. However, much less is known about the DoF of two-way communications. Recently, [24] considered a half-duplex twopair two-way interference channel where nodes other than the relay may not employ interaction and hence are much more restricted than the nodes here (i.e. transmit signals are functions of the messages only and not past outputs). When the relay has 2 antennas, they showed that 4/3 DoF are achievable. No converse results where provided. The authors in [25] studied the DoF of a $K$-pair-user network where pairs of users exchange messages with the help of a MIMO causal relay. All nodes operate in half-duplex mode and there is no direct link between the users. For this setting the full $2 \mathrm{~K}$ DoF may be achieved when the relay has at least $2 K$ antennas. In [26], the authors identified the DoF of the full-duplex 2-pair and 3-pair two-way multi-antenna relay MIMO interference channel, in which again there is no interference between users who only communicate through the relay (no direct links). We consider direct links between all users in the full-duplex bi-directional interference network, as well as links between all users and the relay. We also note that the general results of [19], which state that relays, noisy cooperation, perfect 
feedback and full-duplex operation does not increase the DoF of one-way networks, do not apply, as we consider nodes which are both sources and destinations of messages (two-way rather than one-way). In contrast, work such as [27] on the DoF of two-user wireless networks with instantaneous relays show that when relays are instantaneous rather than causal, DoF gains are to be had. Multi-way relays channels, where clusters of users each wish to exchange messages through the help of a relay have been considered for fairly general classes of channels in [28]-[30]. The framework in [28], [29] would be able to capture our channel (with relay) as a special case if direct links between channels existed; however users are only able to exchange messages through the relays. Of interest in these schemes is the usage of lattice coding and networkcoding based schemes, which have also been used in [31]-[33] to characterize achievable DoF.

The $K$-user interference channel, as an extension of the 2-user interference channel, information theoretically models wireless communications in networks involving more than two pairs of users. Using the idea of interference alignment [18], [34], [35], the DoF of the $K$-user (one-way) IC for both timevarying channels and (almost all) ${ }^{1}$ constant channels has been shown to be $K / 2$ in [18] and [36] respectively. The generalized DoF of the $K$-user IC without and with feedback have been characterized in [37] and [38] (full feedback from receiver $i$ to transmitter $i$ ) respectively. Authors in [39] showed that for almost all constant channel coefficients of fully connected two-hop wireless networks with $K$ sources, $K$ relays and $K$ destinations (source nodes are not destination nodes as they are here, i.e. the network is one-way), the DoF is $K$.

We note that our work differs from prior work in that we consider an interactive, full-duplex Gaussian $K$-pair-user bidirectional interference network for the first time, with and without a relay (which may be either non-causal or causal), and obtain not only sum-rate achievability but also converse DoF results for all three general channel models considered. We emphasize that we seek information theoretic DoF results, which act as benchmarks / upper bounds on the performance of practical systems.

\section{Outline}

We present the system model for the $K$-pair-user full-duplex bi-directional interference network with and without a relay in Section II. We derive a new outer bound to show that $K$ DoF is optimal for the Gaussian $K$-pair-user full-duplex bidirectional interference network in Section III; achievability follows from interference alignment for the non-adaptive oneway $K$-user IC. Then we proceed to consider the $K$-pairuser full-duplex bi-directional interference network with an instantaneous MIMO relay in Section IV, where we show that the maximum $2 K$ DoF may be achieved with the help of an instantaneous MIMO relay with at least $2 K$ antennas. We demonstrate a one-shot achievability scheme. We comment on the possibility of reducing the number of antennas at the instantaneous relay node. In Section $\mathrm{V}$ we then show that if the relay is causal rather than non-causal, that, for the $K$-pair-user

\footnotetext{
${ }^{1}$ The precise definition of "almost all" may be found in [36].
}

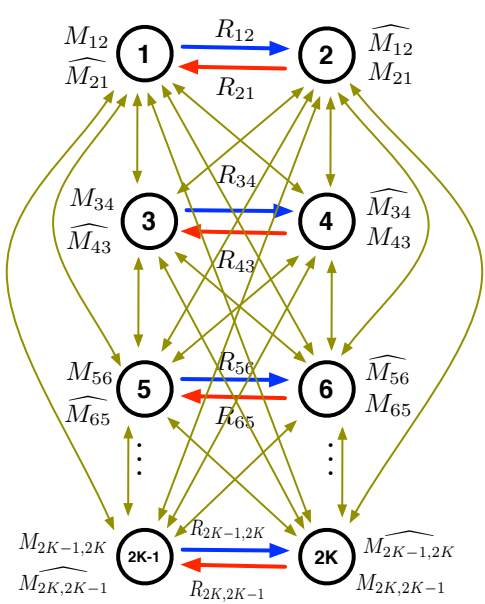

Fig. 1: $K$-pair-user full-duplex bi-directional interference networks. $M_{i j}$ denotes the message known at node $i$ and desired at node $j$ of rate $R_{i j} ; \widehat{M_{i j}}$ denotes that $j$ would like to decode the message $M_{i j}$ from node $i$.

full-duplex bi-directional interference network, the presence of a full-duplex, multi-antenna relay cannot increase the DoF beyond $K$ (which is achievable without relays and without interaction, but requires full-duplex operation). This is done by developing a new outer bound which allows for interaction and causal relaying. We conclude the paper in Section VI.

\section{SySTEM MOdEL}

We describe the $K$-pair-user full-duplex bi-directional interference network without and with a relay in this section.

\section{A. K-pair-user full-duplex bi-directional interference network}

We consider a $K$-pair-user full-duplex bi-directional interference network as shown in Fig. 1, where there are $2 K$ messages and $2 K$ terminals which are all connected to one another. Each of $K$ pairs of users wishes to exchange messages in a bi-directional fashion, while interfering with and experiencing interference from all other pairs in the network. This resembles a $K$-user interference channel (IC) in the $\rightarrow$ direction ( $K$ messages) and another $K$-user IC in the $\leftarrow$ direction ( $K$ messages), where terminals on the same side are connected to each other. All nodes are able to operate in full-duplex mode, i.e. they can transmit and receive signals simultaneously.

At each time slot $k$, the system input/output relationships are described as:

$$
Y_{j}[k]=\sum_{i=1, i \neq j}^{2 K} h_{i j}[k] X_{i}[k]+Z_{j}[k],
$$

where $X_{i}[k], Y_{j}[k], i, j \in\{1,2, \ldots, 2 K\}$ are the inputs and outputs of user $i, j$ at time slot $k$, and $h_{i j}[k], i, j \in\{1,2, \ldots, 2 K\}$ is the channel coefficient from node $i$ to node $j$ at time 
slot $k .^{2}$ The network is subject to complex Gaussian noise $Z_{j}[k] \sim \mathcal{C N}(0,1), j \in\{1,2, \ldots, 2 K\}$ which are independent across users and time slots. We consider time-varying channel coefficients, which for each channel use are all drawn from a continuous distribution (which need not be the same for all channel gains and time instances, as long as they are continuous) and whose absolute values are bounded between a nonzero minimum value and a finite maximum value ${ }^{3}$. Note one can also alternatively consider a frequency selective rather than time-varying system model.

We further assume per user, per symbol power constraints $E\left[\left|X_{i}[k]\right|^{2}\right] \leq P, i \in\{1,2, \ldots, 2 K\}, k \in\{1,2, \cdots n\}$, for block length $n .{ }^{4}$ User $2 i-1$ and $2 i$ wish to exchange messages for $i=1,2, \cdots K$ (user 1 sends to 2,2 to $1, \ldots, 2 K-1$ to $2 K$, $2 K$ to $2 K-1)$ with interactive encoding functions

$$
X_{i}[k]=f\left(M_{i j}, Y_{i}^{k-1}\right), \quad k=1,2, \cdots n
$$

at rate $R_{i, j}=\frac{\log _{2}\left|M_{i j}\right|}{n}$, where $Y_{i}^{k-1}$ denotes the vector $\left(Y_{i}[1], \cdots Y_{i}[k-1]\right)$ from time slot, or channel use 1 to $k-1$ received at user $i$, and $n$ denotes the total number of channel uses (the blocklength). In other words, all users in this network can adapt current channel inputs to previously received channel outputs. The nodes $2 i-1$ and $2 i$ have decoding functions which map $\left(Y_{2 i-1}^{n}, M_{2 i-1,2 i}\right)$ to an estimate of $M_{2 i, 2 i-1}$ and $\left(Y_{2 i}^{n}, M_{2 i, 2 i-1}\right)$ to $M_{2 i-1,2 i}$, respectively. A rate tuple $\left(R_{i, i+1}(P), R_{i+1, i}(P)\right)_{i \in\{1,3, \ldots, 2 K-1\}}$, where we use the argument $P$ simply to remind the reader that this rate is indeed a function of the power constraint $P$, is said to be achievable if there exist a set of interactive encoders and decoders such that the desired messages can be estimated with arbitrarily small probability of error when the number of channel uses $n$ tends to infinity. The sum DoF characterizes the sum capacity of this Gaussian channel at high SNR and is defined as the maximum over all achievable $\left(R_{i, i+1}(P), R_{i+1, i}(P)\right)_{i \in\{1,3, \ldots, 2 K-1\}}$ of

$$
\begin{aligned}
d_{\text {sum }} & =\sum_{i=1,3, \ldots, 2 K-1}\left(d_{i, i+1}+d_{i+1, i}\right) \\
& =\limsup _{P \rightarrow \infty} \frac{\sum_{i=1,3, \ldots, 2 K-1}\left(R_{i, i+1}(P)+R_{i+1, i}(P)\right)}{\log (P)} .
\end{aligned}
$$

Notice the implicit definitions of the DoF of the link from user $i$ to user $i+1, d_{i, i+1}$ and the reverse $d_{i+1, i}$.

\section{B. K-pair-user full-duplex bi-directional interference network with a MIMO relay}

We consider a $K$-pair-user full-duplex bi-directional interference network with a MIMO relay as shown in Fig. 2. All the

\footnotetext{
${ }^{2}$ Note that if user $i$ is transmitting, we have already assumed that its own "self-interference" signal has been ideally subtracted off its received signal, and is hence not present in the above description of channel inputs and outputs. This idealization will of course form an upper bound on what is possible if full self-interference cancellation is not possible, which is outside the scope of this paper and is an interesting topic for future work

${ }^{3}$ The non-zero minimum ensures that the network is fully connected; if certain links are zero/missing DoF results can change dramatically. The finite maximum, besides being realistic practically, also ensures that when we let SNR go to infinity, that all links grow at the same rate, as in the DoF definition.

${ }^{4}$ In our outer bound in Theorem 1, several of the terms may be extended to per user average power constraints, but we leave the per symbol power constraints for simplicity in this initial study, as is often done in degree of freedom results.
}

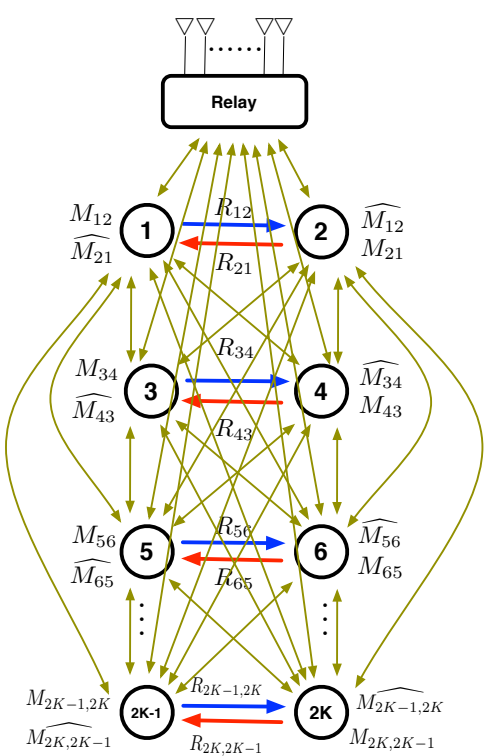

Fig. 2: $K$-pair-user full-duplex bi-directional interference networks with a MIMO relay. $M_{i j}$ denotes the message known at node $i$ and desired at node $j ; \widehat{M_{i j}}$ denotes that $j$ would like to decode the message $M_{i j}$ from node $i$.

system settings are the same as in the previous section except there is a MIMO relay which helps in communicating messages and managing interference in the network. As before, all nodes including the relay are able to operate in full-duplex mode, or transmit and receive at the same time over the same channel, and perfectly cancel out their self-interference.

The relay is assumed to have $M$ antennas and to operate either in a non-causal or "instantaneous" fashion, or in a causal fashion. By "instantaneous" (non causal, relay-without-delay [40]) we refer to its ability to decode and forward signals received at the previous and current (but not future) time slots. We note that this requirement is significantly less strict than a cognitive relay, which would know all users' signals prior to transmission and does not obtain the messages over the air. We will comment more on the usage / impact of a cognitive relay in Section IV-B. Here messages are obtained over the air; the only idealization is the non causality or access to received signals from the current time slot. An alternative motivation for this type of instantaneous relay may be found in [27]. Mathematically, we may describe non causal and causal relaying functions, for each $k=1,2, \cdots n$, as

Non-causal / instantaneous relaying:

$$
\begin{aligned}
& \quad \mathbf{X}_{R}[k]=g_{k}\left(\mathbf{Y}_{R}[1], \mathbf{Y}_{R}[2], \ldots, \mathbf{Y}_{R}[k]\right) \\
& \text { Causal relaying: } \\
& \quad \mathbf{X}_{R}[k]=g_{k}\left(\mathbf{Y}_{R}[1], \mathbf{Y}_{R}[2], \cdots \mathbf{Y}_{R}[k-1]\right),
\end{aligned}
$$

where $\mathbf{X}_{R}[k]$ is a $M \times 1$ ( $M$ antennas) vector signal transmitted by the relay at time slot $k ; g_{k}()$ is a deterministic function; and $\mathbf{Y}_{R}[l], l \in\{1,2, \ldots, k\}$ is the $M \times 1$ vector of signals received at the relay at time slot $l$. The relay is subject to per symbol transmit power constraints over all antennas $E\left[\left\|\mathbf{X}_{R}[k]\right\|_{2}^{2}\right] \leq P_{R}, \forall k \in\{1,2, \cdots n\}$, and global channel 
state information knowledge is assumed at all nodes. At each time slot $k$, the system input/output relationships are:

$$
\begin{aligned}
& Y_{j}[k]=\sum_{i=1, i \neq j}^{2 K} h_{i j}[k] X_{i}[k]+\mathbf{h}_{R j}^{*}[k] \mathbf{X}_{R}[k]+Z_{j}[k], \\
& \mathbf{Y}_{R}[k]=\sum_{i=1}^{2 K} \mathbf{h}_{i R}[k] X_{i}[k]+\mathbf{Z}_{R}[k]
\end{aligned}
$$

where we use the same notation as in (1). In addition, $\mathbf{h}_{i j}[k], i, j \in\{1,2, \ldots, 2 K, R\}$ is the $M \times 1$-dimensional channel coefficient vector from node $i$ to node $j$ at time slot $k$ ( $i$ or $j$ must be the relay node $R$ ), and $\mathbf{Z}_{R}[k] \sim \mathcal{C N}(\mathbf{0}, \mathbf{I})$ is the complex Gaussian noise vector at the relay. The terms in bold represent vectors (due to the MIMO relay). We use * to denote conjugate transpose and ${ }^{T}$ to denote transpose.

\section{Types of signals}

Let $s_{i j}$ denote the independent information symbols (signals) from transmitter $i$ to receiver $j$; these are real numbers which will be combined into the signals $X_{i}[k]$ transmitted by node $i$ at channel use $k$. The received signal at any given node may be broken down into three types of signals:

- the self-interference signal (SI, sent by itself, known to itself);

- the interference signal (sent by the undesired user(s));

- the desired signal (sent by the desired user), respectively. For example, at receiver $1, s_{12}$ is a self-interference signal (SI); $s_{34}, s_{43}, \cdots, s_{2 K-1,2 K}, s_{2 K, 2 K-1}$ are the interference signals; $s_{21}$ is the desired signal. Note we have already removed selfinterference signals from the input/output equations (1)-(2), but SI terms may still be transmitted by the relay (or other users due to adaptation) and hence received.

\section{DOF OF FULL-DUPLEX BI-DIRECTIONAL INTERFERENCE NETWORKS}

In this section we show that the degrees of freedom of the $K$-pair-user full-duplex bi-directional interference networks is $K$, i.e. $K / 2$ in each direction (the DoF of a one-way $K$-user IC is $K / 2$ [18]), for both time-varying and (almost all) constant channel coefficients. In other words, each user still gets $1 / 2$ DoF. The main result of this section is stated in the following theorem:

Theorem 1. The K-pair-user full-duplex bi-directional interference network has $K$ degrees of freedom.

\section{Proof:}

1) Achievability: That $1 / 2$ DoF can be achieved for each transmitter-receiver pair in this network may be seen as follows: our $K$-pair two-way fully connected interference channel may be viewed as a $2 K$ user interference channel where each user interferes with all the others (i.e. each receiver sees $2 K-1$ interfering terms). We may propose an achievability scheme which ignores the ability of nodes to adapt current channel inputs based on past channel outputs, and simply uses the well known interference alignment scheme [18] to, at each decoder, align all the interference other than the self-interference into half of the interference subspace. When the original network is time-varying, i.e., $h_{i j}, i \neq j$ are drawn i.i.d. from a continuous distribution, the achievability follows the scheme in [18], which achieves $1 / 2 \mathrm{DoF}$ per user asymptotically by symbol extension. The key condition that needs to be satisfied for the application of this scheme is the independence between direct links and interfering links, which is true. On the other hand, when the original network is constant, i.e., $h_{i j}, i \neq j$ are arbitrary non-zero constants, $1 / 2$ DoF per transmitter-receiver pair can be achieved by the real alignment scheme [36].

2) Converse: Now we prove the converse, which is valid for both time-varying and constant channel gains. The outer bound may be thought of as a MAC-type bound ${ }^{5}$ often used in oneway interference channels [41], one-way interference channels with generalized feedback [42], two-way interference channels [11], and $K$-user interference channels [43]. In particular, we look at a particular pair of transmitters and corresponding receivers and provide them with all other non-desired messages. For these two users, we then further provide an asymmetric form of side information of the form provided in one of the sum-rates for one-way interference channels [41], which has been extended to two-way interference channels as well as $\mathrm{K}$-user (one-way) interference channels [43] and interference with generalized feedback [42]. We want to remind the reader that all nodes may employ adaptation / interaction and form inputs based on previously received outputs, and that this must hence be allowed in the converse. Hence, our side-information structure is slightly more involved and consists of a larger number of messages, output and noise terms.

Let $M_{A}$ denote all the messages except $M_{12}, M_{34}$, and let $Y_{(3, \ldots, 2 K) \backslash 4}$ denote all outputs except $Y_{1}, Y_{2}, Y_{4}$. Inputs $X_{(3, \ldots, 2 K) \backslash 4}$ and noise term $Z_{(3, \ldots, 2 K) \backslash 4}$ are defined in a similar fashion. $h_{1(3, \ldots, 2 K) \backslash 4}$ denotes the channel gains from user 1 to all the other users except user 2 and 4 . We start by bounding the sum of a pair of rates:

$$
\begin{aligned}
& n\left(R_{12}+R_{34}-\epsilon\right) \\
& \stackrel{(a)}{\leq}\left(M_{34} ; Y_{4}^{n} \mid M_{A}\right)+I\left(M_{12} ; Y_{2}^{n}, Y_{4}^{n}, Y_{(3, \ldots, 2 K) \backslash 4}^{n} \mid M_{34}, M_{A}\right) \\
& =H\left(Y_{4}^{n} \mid M_{A}\right)-H\left(Y_{4}^{n} \mid M_{34}, M_{A}\right) \\
& \quad+H\left(Y_{2}^{n}, Y_{4}^{n}, Y_{(3, \ldots, 2 K) \backslash 4}^{n} \mid M_{34}, M_{A}\right) \\
& \quad-H\left(Y_{2}^{n}, Y_{4}^{n}, Y_{(3, \ldots, 2 K) \backslash 4}^{n} \mid M_{12}, M_{34}, M_{A}\right) \\
& \quad H\left(Y_{4}^{n} \mid M_{A}\right)+H\left(Y_{2}^{n}, Y_{(3, \ldots, 2 K) \backslash 4}^{n} \mid Y_{4}^{n}, M_{34}, M_{A}\right) \\
& \quad-H\left(Y_{2}^{n}, Y_{4}^{n}, Y_{(3, \ldots, 2 K) \backslash 4}^{n} \mid M_{12}, M_{34}, M_{A}\right)
\end{aligned}
$$

where (a) follows as all messages are independent of each other, and we add side information $Y_{4}^{n}, Y_{(3, \ldots, 2 K) \backslash 4}^{n}$ to receiver 2 . Now we bound the three terms above respectively. We start with the first term:

$$
H\left(Y_{4}^{n} \mid M_{A}\right) \leq H\left(Y_{4}^{n}\right) \stackrel{(b)}{\leq} n(\log (P)+o(\log (P)))
$$

where in (b) we have used the fact that Gaussians maximize entropy subject to power constraints (which we recall are $P$ at

\footnotetext{
${ }^{5}$ By MAC-type bound we mean only in the DoF sense, where the sideinformation provided effectively turns the channel into a two-user MAC channel once the noise may be ignored.
} 
each user and time slot). Due to adaptation, the inputs $X_{i}, i \in$ $\{1,2, \ldots, 2 K\}, i \neq 4$ may be correlated, but even if all users are fully correlated and all the transmitters meet the power constraint $P, H\left(Y_{4}^{n}\right) \leq n(\log P+o(\log P))$ as correlation only induces a power gain inside the logarithm for a single antenna receiver. ${ }^{6}$ Here $f(x)=o(\phi(x))$ denotes the Landau little-O notation, i.e. that $\lim _{x \rightarrow \infty} \frac{f(x)}{\phi(x)}=0$.

The second term can be bounded as in (6) - (8) where in step (c) the term $h_{14}[k] X_{1}[k]+Z_{4}[k]$ in the conditioning is decoded from $Y_{4}^{n}$, and then we use conditioning reduces entropy. For simplicity, we use the expression $h_{1(3, \ldots, 2 K) \backslash 4}[k] X_{1}[k]+Z_{(3, \ldots, 2 K) \backslash 4}[k]$ to denote multiple terms $h_{13}[k] X_{1}[k]+Z_{3}[k], h_{15}[k] X_{1}[k]+Z_{5}[k] \ldots$, which is also used in step (d), which follows since Gaussians maximize conditional entropies, as in for example [19, Equation (30)].

Finally, the negative third term can be lower bounded as follows:

$$
\begin{aligned}
& H\left(Y_{2}^{n}, Y_{4}^{n}, Y_{(3, \ldots, 2 K) \backslash 4}^{n} \mid M_{12}, M_{34}, M_{A}\right) \\
& \geq H\left(Y_{2}^{n}, Y_{4}^{n}, Y_{(3, \ldots, 2 K) \backslash 4}^{n} \mid M_{12}, M_{34}, M_{A}, X_{1}^{n}, X_{2}^{n}, \ldots, X_{2 K}^{n}\right) \\
& =H\left(Z_{2}^{n}, Z_{4}^{n}, Z_{(3, \ldots, 2 K) \backslash 4}^{n}\right) \\
& =n \log (2 \pi e)^{2 K-1} \\
& =n(O(1)),
\end{aligned}
$$

where $f(x)=O(\phi(x))$ denotes that $|f(x)|<A \phi(x)$ for some constant $A$ and all values of $x$. Now combining everything, and taking the limit,

$$
d_{12}+d_{34} \leq \limsup _{P \rightarrow \infty} \frac{R_{12}+R_{34}}{\log (P)}=1+0+0-0=1
$$

From the above we see that the DoF per pair of users transmitting in the same direction is 1 . Summing over all rate pairs leads to the theorem.

Remark 1. In the derived outer bound we considered interaction between users, i.e. $X_{i}[k]=f\left(M_{i j}, Y_{i}^{k-1}\right)$. However, as we showed, this does not help a user to achieve more than 1/2 DoF. This may be intuitively explained as follows: 1) the DoF measures the number of clean information streams that may be transmitted at high SNR when the desired signals and interference signals are received roughly "at the same level" (SNR and INR scale to infinity at the same rate). In this case, rates cannot be improved by having users send messages of other users to re-route the message (i.e. message from user 1 to 2 could go via another user rather than the direct link) as all links are equally strong. One would thus need to tradeoff one's own rate to relay another user's rate given the symmetry in the channels. 2) Adaptation allows for the correlation of messages at transmitters. In Gaussian channels, such correlation may be translated into coherent power gains inside the logarithm. The DoF metric is insensitive to coherent power gains as it measures pre-logarithm gains, not constant power factor gains inside the logarithm.

Remark 2. Note if $K$ is an odd number, we can sum over the rate pairs on the same side of the network in a cyclic way

\footnotetext{
${ }^{6}$ We note that a bound of $n \log (P)+o(\log (P))$ may also be shown to hold for average rather than per symbol power constraints of $P$ at each transmitter using Jensen's inequality and using that $2 \sqrt{P_{i} P_{j}} \leq P_{i}+P_{j}$.
}

and then divide by 2 to prove the converse. Let's take $K=3$ as an example. In this case, we can prove $d_{12}+d_{34} \leq 1$, $d_{12}+d_{56} \leq 1$ and $d_{34}+d_{56} \leq 1$ by following the same steps in proving (10). Then we sum over these pairs and divide by 2 we have $\left[2\left(d_{12}+d_{43}+d_{56}\right)\right] / 2 \leq 3 / 2$. Similarly for the opposite direction, and thus $d_{\text {sum }} \leq 3$.

\section{DOF OF FULL-DUPLEX BI-DIRECTIONAL INTERFERENCE NETWORKS WITH AN INSTANTANEOUS MIMO RELAY}

In this section, we obtain the DoF of the $K$-pair-user fullduplex bi-directional interference network with an instantaneous MIMO relay with $M=2 K$ antennas in the system model described in Section II-B. We then make a number of comments on how to reduce the number of antennas at the relay, at the expense of for example diminished achievable degrees of freedom, or requiring partial cognition of the messages at the relay.

A. DoF of $K$-pair-user full-duplex bi-directional interference network with an instantaneous $2 K$-antenna Relay

We show our second main result: that the maximum $2 K$ DoF of the $K$-pair-user full-duplex bi-directional interference network with an instantaneous $2 K$-antenna relay is achievable.

Theorem 2. The K-pair-user full-duplex bi-directional interference networks with an instantaneous $2 K$-antenna relay has $2 K$ degrees of freedom.

Proof:

1) Converse: The converse is trivial since for a $2 K$ user, $2 K$ message unicast network where all sources and destinations have a single antenna, the maximum degrees of freedom cannot exceed $2 K$ by cut-set arguments, even with adaptation/interaction at all nodes.

2) Achievability: We propose a simple "one-shot" scheme. We consider the Gaussian channel model at high SNR, and hence noise terms are ignored from now on.

The $2 K$ users each transmit a symbol $s_{i j}$ (from user $i$ to user $j$ ) and the relay receives:

$$
\mathbf{Y}_{R}=\sum_{i=1}^{2 K} \mathbf{h}_{i R} s_{i j}, \text { for the appropriate } j \text { values, see Fig. } 2 .
$$

The $2 K$-antenna relay (with global CSI) decodes all $2 K$ symbols using a zero-forcing decoder [44], and due to the instantaneous property, transmits the following signal in the same time slot:

$$
\mathbf{X}_{R}=\sum_{i=1}^{2 K} \mathbf{u}_{i j} s_{i j}
$$

where $\mathbf{u}_{i j}$ denote the $2 K \times 1$ beamforming vectors carrying signals from user $i$ to intended user $j$. Now at receiver 1 (for example),

$$
Y_{1}=\sum_{i=2}^{2 K} h_{i 1} s_{i j}+\mathbf{h}_{R 1}^{*} \mathbf{X}_{R}, \text { for the appropriate } j \text { values. }
$$




$$
\begin{aligned}
& H\left(Y_{2}^{n}, Y_{(3, \ldots, 2 K) \backslash 4}^{n} \mid Y_{4}^{n}, M_{34}, M_{A}\right) \\
& =\sum_{k=1}^{n}\left[H\left(Y_{2}[k], Y_{(3, \ldots, 2 K) \backslash 4}[k] \mid Y_{2}^{k-1}, Y_{(3, \ldots, 2 K) \backslash 4}^{k-1}, Y_{4}^{n}, M_{34}, M_{A}, X_{2}^{k}, X_{(3, \ldots, 2 K) \backslash 4}^{k}, X_{4}^{n}\right]\right. \\
& \stackrel{(c)}{\leq} \sum_{k=1}^{n}\left[H\left(h_{12}[k] X_{1}[k]+Z_{2}[k], h_{1(3, \ldots, 2 K) \backslash 4}[k] X_{1}[k]+Z_{(3, \ldots, 2 K) \backslash 4}[k] \mid h_{14}[k] X_{1}[k]+Z_{4}[k]\right)\right] \\
& \stackrel{(d)}{\leq} \sum_{k=1}^{n}\left[\log 2 \pi e\left(1+\frac{h_{12}^{2}[k] P}{1+h_{14}^{2}[k] P}\right)+\log 2 \pi e\left(1+\frac{h_{1(3, \ldots, 2 K) \backslash 4}^{2}[k] P}{1+h_{14}^{2}[k] P}\right)\right] \\
& =n(o(\log (P)))
\end{aligned}
$$

At receiver 1, the interference signals received from the relay are used to neutralize the interference signals received from the transmitters. To do this, we design the beamforming vectors to satisfy:

$h_{i 1}+\mathbf{h}_{R 1}^{*} \mathbf{u}_{i j}=0, \quad i=3,4, \ldots, 2 K$, for appropriate $j$ values

The $2 K \times 1$ beamforming vectors satisfying the needed constraints always exist, by a dimensionality argument, along with the random channel coefficients. To see this, take $\mathbf{u}_{34}$ as an example. We wish to construct $\mathbf{u}_{34}$ such that the following conditions are satisfied:

$$
h_{3 j}+\mathbf{h}_{R j}^{*} \mathbf{u}_{34}=0, \quad j=1,2, \ldots, 2 K \text { except } 3,4 .
$$

The $2 K$ dimensional beamforming vector $\mathbf{u}_{34}$ has $2 K$ free parameters, which are reduced to 2 in order to satisfy the $2 K-2$ conditions in (13). That is, $2 K-(2 K-2)=2$. Thus, let $a, b$ be two scalars, let $\mathbf{A}, \mathbf{B}$ be $1 \times 2 K$ vectors such that the matrix below is invertible, then the following choice of beam forming vector (for example) will satisfy all conditions:

$$
\mathbf{u}_{34}=\left[\begin{array}{c}
\mathbf{h}_{R 1}^{*} \\
\mathbf{h}_{R 2}^{*} \\
\mathbf{h}_{R 5}^{*} \\
\mathbf{h}_{R 6}^{*} \\
\vdots \\
\mathbf{h}_{R, 2 K}^{*} \\
\mathbf{A} \\
\mathbf{B}
\end{array}\right]^{-1}\left[\begin{array}{c}
-h_{31} \\
-h_{32} \\
-h_{35} \\
-h_{36} \\
\vdots \\
-h_{3,2 K} \\
a \\
b
\end{array}\right] .
$$

Note that all the beam forming vectors must also be chosen to satisfy the relay power constraint $P_{R}$, but that we have sufficient degrees of freedom (choices of $a, b)$ to ensure this, and that this will not affect the DoF in either case, as we will let $P_{R} \rightarrow \infty$.

Still at receiver 1 , once the interference signals have been neutralized and the self-interference (SI) signal $s_{12}$ has been subtracted off, the received signal in (11) becomes

$$
Y_{1}-S I=h_{21} s_{21}+\mathbf{h}_{R 1}^{*} \mathbf{u}_{21} s_{21},
$$

from which the desired signal $s_{21}$ can be easily decoded as long as $h_{21} \neq-\mathbf{h}_{R 1}^{*} \mathbf{u}_{21}$, which we may guarantee by proper scaling of $\mathbf{u}_{21}$. Similar decoding is performed at all other receivers.
Remark 3. To achieve $2 K$ DoF we have assumed full duplex operation. If instead all nodes operate in half-duplex mode, it is trivial to achieve half the full-duplex DoF, i.e. to achieve $K$ DoF. To do so, in the first time slot, all $2 K$ users transmit a message, and the $2 K$-antenna relay listens and decodes all $2 K$ messages using a zero-forcing decoder. At time slot 2 , the relay broadcasts a signal and all users listen. By careful choice of beamforming vectors as in (14), for example, each receiver receives only their desired message in this time slot. Therefore $2 K$ desired messages are obtained in 2 time slots, i.e. $K$ DoF is achievable. Note however that in the half-duplex setting, the relay is causal rather than non-causal or instantaneous.

Remark 4. We have shown in the previous section that the DoF of the K-pair-user full-duplex bi-directional interference networks is $K$; Theorem 2 implies that the addition of an instantaneous $2 K$-antenna relay can increase the DoF of the $K$-pair-user full-duplex bi-directional interference networks to $2 K$ - it essentially cancels out all interference at all nodes simultaneously. Note this DoF increase is due to non-causal relaying rather than interaction between users.

B. Comments on reducing the number of antennas at the instantaneous relay

We now investigate the achievable DoF using a reduced number of antennas at the instantaneous relay. For simplicity, we first consider the (2-pair-user) full-duplex bi-directional interference networks with an instantaneous 3-antenna relay (the general $K$-pair-user networks with a $(2 K-1)$-antenna relay is a simple extension of this case), for which we propose another linear one-shot strategy to achieve $3 \mathrm{DoF}$. Whether this is the optimal achievable DoF is still open, i.e. unlike in all other sections so far, we have not obtained a converse (whether anything between 3 and the maximal $4 \mathrm{DoF}$ is achievable is left open).

Theorem 3. For the (2-pair-user) full-duplex bi-directional interference networks with an instantaneous 3-antenna relay, 3 degrees of freedom are achievable.

Proof: The proof of this theorem is similar to the "oneshot" scheme in the previous section and is thus omitted here.

If we further 1) restrict the relay to be linear, i.e. to be of 


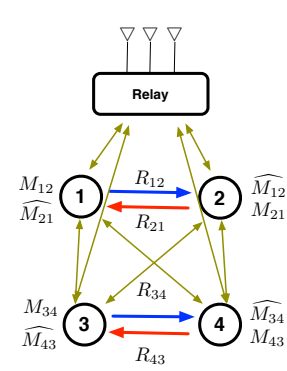

(a) 2-pair-user full-duplex bi-directional interference networks with instantaneous 3 -antenna relay.

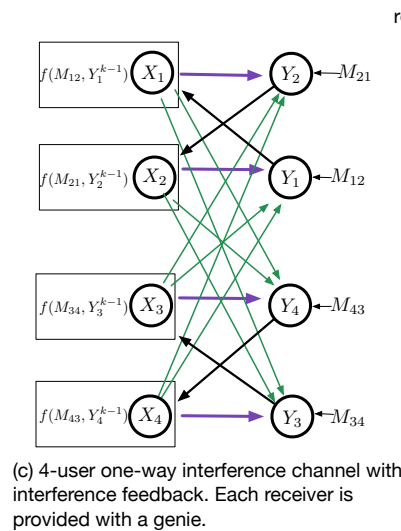

Fig. 3: Network transforms

the form

$$
\mathbf{X}_{R}[k]=\mathbf{A} \mathbf{Y}_{R}^{k}
$$

where $\mathbf{A}$ is a $3 \times 3 k$ matrix (3-antenna), and 2) we prohibit time extension i.e. limit the block length to $n=1$, then we can show that $3 \mathrm{DoF}$ is the maximal achievable as well, i.e. the DoF under these restrictions is exactly 3.

Theorem 4. For the (2-pair-user) full-duplex bi-directional interference networks with an instantaneous 3-antenna relay which is constrained to using a one-shot (no symbol extensions) linear beamforming scheme, the DoF is 3.

Proof: The channel model of Theorem 3 is shown in Fig.3(a), and we recall that for $i=1,2,3,4$ and proper $j$,

$$
X_{i}[k]=f\left(M_{i j}, Y_{i}^{k-1}\right), \quad k=1,2, \cdots n .
$$

For the instantaneous 3-antenna relay with linear relaying, we have

$$
\mathbf{X}_{R}[k]=g_{k}\left(\mathbf{Y}_{R}^{k}\right)=\mathbf{A} \mathbf{Y}_{R}^{k},
$$

where $\mathbf{A}$ is a $3 \times 3 k$ matrix.

We now perform two transformations which do not affect the DoF of the network, followed by a third which may enlarge the DoF. The original and sequence of transformed networks is shown in Fig. 3. In the first, we represent the two-pairuser bi-directional interference network with an instantaneous relay as a four-user one-way interference channel with an instantaneous relay and causal interference feedback (in black), shown in Fig. 3(b). Note here the feedback (in black) is from the undesired receiver, which is different from the more commonly used feedback from the desired receiver. Also note that the self-interference from the original transmitters has been subtracted off at each receiver (e.g. no link in Fig. 3(b) from Tx 2 to $\operatorname{Rx} 2$ ), but it can still observe self-interference from the instantaneous relay.

Under the assumption of linear operation at the relay, the channel input-output relations are:

$$
\begin{aligned}
& \mathbf{Y}_{R}[k]= \sum_{i=1}^{4} \mathbf{h}_{i R}[k] X_{i}[k]+\mathbf{Z}_{R}[k] \\
& Y_{j}[k]=\sum_{i=1, i \neq j}^{4} h_{i j}[k] X_{i}[k]+\mathbf{h}_{R j}^{*}[k] \mathbf{X}_{R}[k]+Z_{j}[k] \\
&=\sum_{i=1, i \neq j}^{4} h_{i j}[k] X_{i}[k]+\mathbf{h}_{R j}^{*}[k] \mathbf{A}\left(\sum_{i=1}^{4} \mathbf{h}_{i R}^{k} X_{i}^{k}+\mathbf{Z}_{R}^{k}\right)+Z_{j}[k] \\
&=\sum_{i=1, i \neq j}^{4}\left(\left(h_{i j}[k]+\mathbf{h}_{R j}^{*}[k] \mathbf{A}^{*} \mathbf{h}_{i R}[k]\right) X_{i}[k]+\mathbf{h}_{R j}^{*}[k] \mathbf{A}^{\prime} \mathbf{h}_{i R}^{k-1} X_{i}^{k-1}\right) \\
& \quad+\mathbf{h}_{R j}^{*}[k] \mathbf{A} \mathbf{h}_{j R}^{k} X_{j}^{k}+\mathbf{h}_{R j}^{*}[k] \mathbf{A} \mathbf{Z}_{R}^{k}+Z_{j}[k] \\
&=\sum_{i=1, i \neq j}^{4}\left(\left(h_{i j}[k]+\mathbf{h}_{R j}^{*}[k] \mathbf{A}^{*} \mathbf{h}_{i R}[k]\right) X_{i}[k]+\mathbf{h}_{R j}^{*}[k] \mathbf{A}^{\prime} \mathbf{h}_{i R}^{k-1} X_{i}^{k-1}\right) \\
& \quad+\mathbf{h}_{R j}^{*}[k] \mathbf{A} \mathbf{Z}_{R}^{k}+Z_{j}[k] \\
& \triangleq \sum_{i=1, i \neq j}^{4}\left(g_{i j}[k] X_{i}[k]+\mathbf{h}_{R j}^{*}[k] \mathbf{A}^{\prime} \mathbf{h}_{i R}^{k-1} X_{i}^{k-1}\right) \\
&+\mathbf{h}_{R j}^{*}[k] \mathbf{A} \mathbf{Z}_{R}^{k}+Z_{j}[k]
\end{aligned}
$$

where $\mathbf{A}^{*}$ is the first 3 columns of $\mathbf{A}, \mathbf{A}^{\prime}$ is all but the first 3 columns of $\mathbf{A}$. We drop the self-interference term $\mathbf{h}_{R j}^{*}[k] \mathbf{A} \mathbf{h}_{j R}^{k} X_{j}^{k}$ in (18) since $M_{j}$ is provided to receiver $j$, which can therefore construct $X_{j}^{k}$ and subtract it. Now incorporate the instantaneous relay into the channel (second network transform), as shown in Fig. 3(c). Note that we used different colors for the channel links since the channel is different compared to that in Fig. 3(b). Since we have prohibited time extensions, we consider a one shot scheme, in which case $\mathbf{A}=\mathbf{A}^{*}$ is of size $3 \times 3$ and $\mathbf{A}^{\prime}=\emptyset$. Thus (20) becomes (omit the noise terms and time index for simplicity, and recall that $g_{i j}$ is defined in (20))

$$
Y_{j}=\sum_{i=1, i \neq j}^{4} g_{i j} X_{i}
$$

In a converse, each receiver can successfully decode the desired message with probability 1 . Let receiver 1 and 2 cooperate (act as 1 receiver with 2 antennas) - which cannot reduce the sum DoF - and subtract the decoded messages from their received signals. Now we observe

$$
\left[\begin{array}{l}
Y_{1} \\
Y_{2}
\end{array}\right]=\left[\begin{array}{ll}
g_{31} & g_{41} \\
g_{32} & g_{42}
\end{array}\right]\left[\begin{array}{c}
X_{3} \\
X_{4}
\end{array}\right] \triangleq G_{(34)(12)}\left[\begin{array}{c}
X_{3} \\
X_{4}
\end{array}\right]
$$


in Fig. 3(d). Inspired by and following the same techniques used in [45], we claim that by providing an additional $2-$ $\operatorname{rank}\left(G_{(34)(12)}\right)$ antennas (random linear combinations) to the cooperating receivers 1 and 2 (top circled receiver in Fig. 3(d)), they can decode $X_{3}$ and $X_{4}$. Thus we now have a MAC channel with four transmitters and one $\left(2+2-\operatorname{rank}\left(G_{(34)(12)}\right)\right)$ antenna receiver ${ }^{7}$. The cut-set bound for this MAC channel yields

$$
D o F_{\text {sum }} \leq 2+2-\operatorname{rank}\left(G_{(34)(12)}\right)=4-\operatorname{rank}\left(G_{(34)(12)}\right) .
$$

Similarly letting receiver 3 and 4 cooperate yields

$$
D_{o} F_{\text {sum }} \leq 4-\operatorname{rank}\left(G_{(12)(34)}\right) \text {. }
$$

Taking the minimum of the two bounds yields:

$$
D_{o} F_{\text {sum }} \leq 4-\max \left\{\operatorname{rank}\left(G_{(34)(12)}, \operatorname{rank}\left(G_{(12)(34)}\right)\right\} .\right.
$$

To lower bound the max term, we turn to the technique used in [46]. In [46], an outer bound for the DoF of the 2-user MIMO interference channel with an instantaneous, linear relay is studied. The converse in [46, Sec. IV] includes a step in which the sum of the rank of two cross link channel matrices is lower bounded. Note that

$$
G_{(a b)(c d)}=\left[\begin{array}{ll}
h_{a c} & h_{b c} \\
h_{a d} & h_{b d}
\end{array}\right]+\left[\begin{array}{l}
\mathbf{h}_{R c} \\
\mathbf{h}_{R d}
\end{array}\right] \mathbf{A}\left[\begin{array}{ll}
\mathbf{h}_{a R} & \mathbf{h}_{b R}
\end{array}\right]
$$

where $(a b)(c d)=(12)(34)$ or $(34)(12)$ and all $h$ 's are generic channel coefficients drawn i.i.d. from a continuous distribution. This is in the same form as the channel coefficient matrices of the cross links of the 2-user MIMO interference channel with an instantaneous relay in [46], when the transmitter and receiver have 2 antennas and the relay has 3 antennas. Thus the technique for lower bounding the sum of the rank of two cross link channel matrices in [46] can be applied here to lower bound the max term as

$$
\begin{aligned}
& \max \left\{\operatorname{rank}\left(G_{(34)(12)}, \operatorname{rank}\left(G_{(12)(34)}\right)\right\}\right. \\
& \geq\left(\operatorname{rank}\left(G_{(34)(12)}\right)+\operatorname{rank}\left(G_{(12)(34)}\right)\right) / 2 \\
& \geq \frac{2 \times \# \text { of rows in } G-\# \text { of rows in } \mathbf{A}}{2} \\
& =\frac{4-3}{2}=1 / 2 .
\end{aligned}
$$

where (26) follows the result in [46, Sec. IV.B.5]. This shows that the max term cannot be 0 . Since the rank must be an integer, if no time extension is considered, the max term is lower bounded by 1 . Applying it to (25) we have that the sum DoF is upper bounded by 3 , which shows that our proposed one-shot achievability is optimal.

The above results demonstrates that by reducing the number of antennas at the instantaneous relay from 4 to 3 , we have also reduced the achievable DoF from 4 to 3 . One may ask how else we might be able to reduce the number of antennas without decreasing the DoF. One way is to trade cognition for antennas, as we remark on next.

\footnotetext{
${ }^{7}$ There is some partial feedback in the MAC - the black lines - but this does not affect the DoF.
}

Remark 5. If we consider a cognitive relay (cognitive in the sense of having a-priori knowledge of messages, as first introduced in [47]), which would have access to all 4 users' signals prior to transmission, the number of antennas at the relay can be reduced to 2, while still being able to achieve the maximum 4 degrees of freedom for the (2-pair-user) full-duplex bi-directional interference networks. The achievability scheme is trivial: the cognitive relay broadcasts all 4 signals (desired for each user) and all users listen. By careful choice of the four $2 \times 1$ beamforming vectors to cancel interference signals, and subtracting the self-interference signal, each receiver is able to obtain the desired signal. Therefore the maximal 4 DoF are achieved.

Remark 6. We can do even better: if the cognitive relay only knows 2 users' signals, then we are still able to achieve the maximum 4 DoF with 2 antennas at the relay by a simple oneshot scheme. For example, assume user 1 and 2's signals are known at the relay prior to transmission (knowing any 2 of the 4 messages suffices). Now, each transmitter sends a message $s_{i j}$ and the relay receives 4 messages. Then the 2-antenna relay first subtracts transmitter 1 and 2's messages and uses a zero-forcing decoder to decode the other two messages, and transmits

$$
\mathbf{X}_{R}=\mathbf{u}_{12} s_{12}+\mathbf{u}_{21} s_{21}+\mathbf{u}_{34} s_{34}+\mathbf{u}_{43} s_{43} .
$$

At receiver 1 (for example):

$$
\begin{aligned}
Y_{1} & =h_{21} s_{21}+h_{41} s_{43}+h_{31} s_{34} \\
& +\mathbf{h}_{R 1}^{*} \mathbf{u}_{21} s_{21}+\mathbf{h}_{R 1}^{*} \mathbf{u}_{43} s_{43}+\mathbf{h}_{R 1}^{*} \mathbf{u}_{12} s_{12}+\mathbf{h}_{R 1}^{*} \mathbf{u}_{34} s_{34} .
\end{aligned}
$$

To decode the desired message $s_{21}$, we subtract off the self-interference signal $s_{12}$; neutralize interference signals $s_{34}, s_{43}$ by designing the beamforming vectors such that $h_{31}+\mathbf{h}_{R 1}^{*} \mathbf{u}_{34}=0, h_{41}+\mathbf{h}_{R 1}^{*} \mathbf{u}_{43}=0$. A similar decoding procedure follows for the other receivers, where we note the $2 \times 1$ beamforming vectors can be always constructed by the 2-antenna relay. Therefore, each user is able to get 1 desired signal in 1 time slot and the maximal 4 DoF are achieved.

\section{DOF OF FULL-DUPLEX BI-DIRECTIONAL INTERFERENCE NETWORK WITH A CAUSAL MIMO RELAY}

It is known that for one-way channels where nodes are either sources or destinations of messages but not both as in a two-way setting, the usage of feedback, causal relays (possibly with multiple antennas), and cooperation does not increase the DoF of the network [19]. In the previous section, we showed that a non-causal / instantaneous multi-antenna relay may increase the DoF of a $K$-pair user full-duplex bidirectional interference network to its maximal value of $2 K$ (provided we have sufficient number of antennas). Here we show that, in sharp contrast, if the relay is actually causal, it does not increase the DoF of the $K$-pair-user full-duplex bi-directional interference network beyond that of a network without the relay present, which would have $K \operatorname{DoF}(K / 2$ in each direction). Intuitively this is because a causal relay cannot mitigate the current interference signals. This result aligns with the one-way results in [19] in the sense that causal relays again 


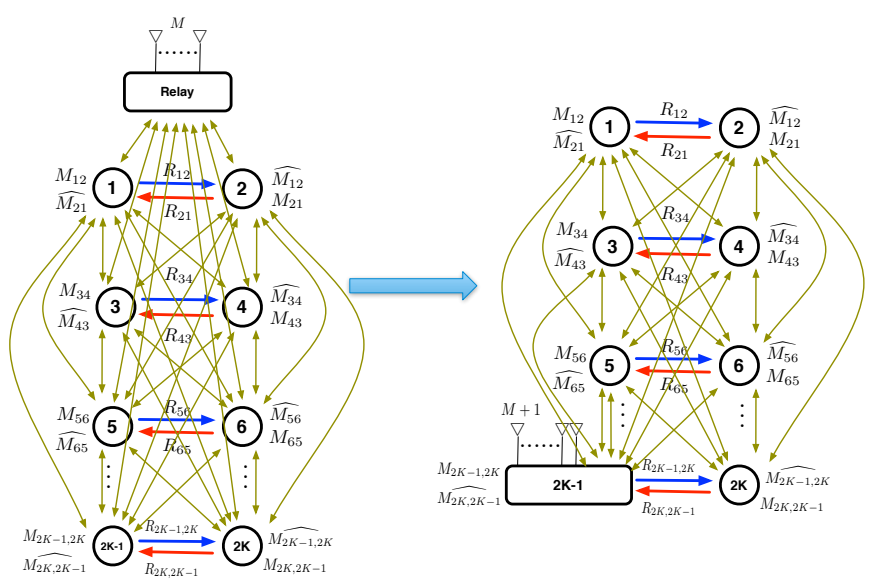

Fig. 4: Transformation of the K-pair-user full-duplex bidirectional interference network.

do not help. However, we note that full-duplex operation does increase the DoF for the bi-directional interference networks in this paper, but does not for their one-way counterparts [19].

We thus consider the $K$-pair-user full-duplex bi-directional interference network with one causal MIMO relay which has $M$ antennas. The system model is the same as that in Section II-B, where we recall that the relay is now causal, and hence

$$
\mathbf{X}_{R}[k]=g_{k}\left(\mathbf{Y}_{R}[1], \mathbf{Y}_{R}[2], \cdots \mathbf{Y}_{R}[k-1]\right),
$$

where $\mathbf{X}_{R}[k]$ is an $M \times 1$ vector signal transmitted by the relay at time $k, g_{k}()$ are deterministic functions for each $k=$ $1,2, \cdots n$, and $\mathbf{Y}_{R}[k]$ is the $M \times 1$ vector of signals received by the relay at time slot $k$. Let $P=P_{R}$ for simplicity (we simply need $P$ and $P_{R}$ to scale to infinity at the same rate). Our third main result is the following.

Theorem 5. The DoF of the K-pair-user full-duplex bidirectional interference network with a causal MIMO relay is $K$.

Proof: Achievability follows from the fact that the DoF of the $K$-pair-user full-duplex bi-directional interference network without a relay is $K$, as shown in Section III.

Now we prove the converse. While the result on DoF for networks with feedback, causal relays, cooperation and fullduplex operation of [19] do not apply here directly as we have nodes which are both sources and destinations of messages, our proof technique is based on the ideas seen in $[19$, Sec. IIIA and IIIB]. In particular, in [19, Sec. IIIA] a network transformation is performed on the $S \times R \times D$ network ( $S$ source nodes, $R$, relay nodes and $D$ destination nodes) rendering it a four node $X$ channel: all relays and all but one source node (which is kept separate) are grouped and permitted to fully cooperate, forming a multi-antenna source node, and all destination nodes but one (which is kept separate) are grouped and permitted to cooperate, forming a multi-antenna destination node. We do something similar but do not reduce our network to 4 nodes, but rather reduce it from $2 K+1$ nodes to $2 K$ nodes as shown in Fig. 4 and then consider a MAC-type outer-bound on pairs of rates.
Since cooperation between nodes cannot reduce the DoF, we let the causal MIMO relay fully cooperate with one of the users, take user $2 K-1$ WLOG. In other words, we co-locate user $2 K-1$ and the relay or put infinite capacity links between these nodes. Then the capacity region of the original network is outer bounded by that of the following $2 K$-node network in which all nodes but node $2 K-1$ have one antenna and one message, and desire one message, and in which node $2 K-1$ has $M+1$ antennas, still only desires message $M_{2 K, 2 K-1}$ and has message $M_{2 K-1,2 K}$ (but may decode other messages as it has multiple antennas).

As done in [19], we more rigorously define the transformed network next. Letting the tilde $\tilde{A}$ notation denote the inputs, outputs and channel gains of the new network, we have the correspondences (or equivalences $\equiv$ for inputs, since they may actually be different due to interaction based on different received signals)

$$
\begin{aligned}
& \tilde{X}_{i} \equiv X_{i}, \quad i=1,2, \ldots, 2 K, \text { except } 2 K-1, \quad \tilde{\mathbf{X}}_{2 K-1}^{T} \equiv\left[X_{2 K-1}, \mathbf{X}_{R}^{T}\right] \\
& \tilde{Z}_{i} \equiv Z_{i}, \quad i=1,2, \ldots, 2 K, \text { except } 2 K-1, \quad \tilde{\mathbf{Z}}_{2 K-1}^{T} \equiv\left[Z_{2 K-1}, \mathbf{Z}_{R}^{T}\right], \\
& \tilde{h_{i j}}=h_{i j}, \quad \text { for appropriate } i, j \text { and } i, j \neq 2 K-1 \\
& \tilde{\mathbf{h}}_{i, 2 K-1}^{T}=\left[h_{i, 2 K-1}, \mathbf{h}_{i R}^{T}\right], \quad i=1,2, \ldots, 2 K, \text { except } 2 K-1, \\
& \tilde{\mathbf{h}}_{2 K-1, j}^{T}=\left[h_{2 K-1, j}, \mathbf{h}_{R j}^{T}\right], \quad j=1,2, \ldots, 2 K, \text { except } 2 K-1,
\end{aligned}
$$

and the following input/output relationships at each channel use, for $j \in\{1,2, \ldots, 2 K\}$ :

$$
\begin{aligned}
& \tilde{Y}_{j}[k]=\sum_{i=1, i \neq j, 2 K-1}^{2 K} \tilde{h}_{i j}[k] \tilde{X}_{i}[k]+\tilde{\mathbf{h}}_{2 K-1, j}^{*}[k] \tilde{\mathbf{X}}_{2 K-1}[k]+\tilde{Z}_{j}[k], \\
& \tilde{\mathbf{Y}}_{2 K-1}[k]=\sum_{i=1, i \neq 2 K-1}^{2 K} \tilde{\mathbf{h}}_{i, 2 K-1}[k] \tilde{X}_{i}[k]+\tilde{\mathbf{Z}}_{2 K-1}[k] .
\end{aligned}
$$

We have the interactive encoding functions at each node

$$
\begin{aligned}
& \tilde{X}_{i}[k]=\tilde{f}_{i}\left(M_{i j}, \tilde{Y}_{i}^{k-1}\right), \quad i=1,2, \ldots, 2 K, \text { except } 2 K-1 \\
& \tilde{\mathbf{X}}_{2 K-1}[k]=\tilde{f}_{2 K-1}\left(M_{2 K-1,2 K}, \tilde{\mathbf{Y}}_{2 K-1}^{k-1}\right)
\end{aligned}
$$

where (29) is where the causality of the relay is observed / incorporated.

Let $M_{A}$ denote all messages except $M_{12}, M_{34}$, and let $\tilde{Y}_{(2, \ldots, 2 K) \backslash 4}$ denote $\tilde{Y}_{2}, \tilde{Y}_{3}, \tilde{Y}_{5}, \ldots, \tilde{Y}_{2 K}$ i.e. all outputs except $\tilde{Y}_{1}$ and $\tilde{Y}_{4}$. Note $\tilde{Y}_{(2, \ldots, 2 K) \backslash 4}$ includes the outputs vector $\tilde{\mathbf{Y}}_{2 K-1}$ at user $2 K-1$. Similarly, $\tilde{X}_{(2, \ldots, 2 K) \backslash 4}$ and $\tilde{Z}_{(2, \ldots, 2 K) \backslash 4}$ denote all inputs / noises except those at nodes 1 and 4.

We now bound the sum-rate in each direction, considering the sum of a pair of rates, we obtain the following MACtype bound, similar to that in Theorem 1. While the type of side-information required is conceptually similar to that of the transformed network in [19] and many outer bounds for interference channels with and without feedback, once again, the structure is slightly different as we have a different transformed model, and as we permit adaptation at all nodes. 
Starting with Fano's inequality, we will have

$$
\begin{aligned}
& n\left(R_{12}+R_{34}-\epsilon\right) \\
& \leq I\left(M_{34} ; \tilde{Y}_{4}^{n} \mid M_{A}\right)+I\left(M_{12} ; \tilde{Y}_{4}^{n}, \tilde{Y}_{(2, \ldots, 2 K) \backslash 4}^{n} \mid M_{34}, M_{A}\right) \\
& =H\left(\tilde{Y}_{4}^{n} \mid M_{A}\right)-H\left(\tilde{Y}_{4}^{n} \mid M_{34}, M_{A}\right) \\
& +H\left(\tilde{Y}_{4}^{n}, \tilde{Y}_{(2, \ldots, 2 K) \backslash 4}^{n} \mid M_{34}, M_{A}\right) \\
& -H\left(\tilde{Y}_{4}^{n}, \tilde{Y}_{(2, \ldots, 2 K) \backslash 4}^{n} \mid M_{34}, M_{A}, M_{12}\right) \\
& \leq H\left(\tilde{Y}_{4}^{n} \mid M_{A}\right)+H\left(\tilde{Y}_{(2, \ldots, 2 K) \backslash 4}^{n} \mid \tilde{Y}_{4}^{n}, M_{34}, M_{A}\right) \\
& -H\left(\tilde{Z}_{4}^{n}, \tilde{Z}_{(2, \ldots, 2 K) \backslash 4}^{n}\right) \\
& =H\left(\tilde{Y}_{4}^{n} \mid M_{A}\right)-H\left(\tilde{Z}_{4}^{n}\right)+H\left(\tilde{Y}_{(2, \ldots, 2 K) \backslash 4}^{n} \mid M_{34}, M_{A}, \tilde{Y}_{4}^{n}\right) \\
& -H\left(\tilde{Z}_{(2, \ldots, 2 K) \backslash 4}^{n}\right) \\
& \leq \sum_{k=1}^{n}\left[H\left(\tilde{Y}_{4}[k]\right)-H\left(\tilde{Z}_{4}[k]\right)\right. \\
& +H\left(\tilde{Y}_{(2, \ldots, 2 K) \backslash 4}[k] \mid \tilde{Y}_{(2, \ldots, 2 K) \backslash 4}^{k-1}, M_{34}, M_{A}, \tilde{Y}_{4}^{n}, \tilde{X}_{4}^{n}, \tilde{X}_{(2, \ldots}^{k}\right. \\
& \left.-H\left(\tilde{Z}_{(2, \ldots, 2 K) \backslash 4}[k]\right)\right] \\
& \leq n(\log P+o(\log P)) \\
& +\sum_{k=1}^{n}\left[H \left(\tilde{h}_{12}[k] \tilde{X}_{1}[k]+\tilde{Z}_{2}[k], \tilde{h}_{13}[k] \tilde{X}_{1}[k]+\tilde{Z}_{3}[k], \cdots,\right.\right. \\
& \tilde{\mathbf{h}}_{1,2 K-1}[k] \tilde{X}_{1}[k]+\tilde{\mathbf{Z}}_{2 K-1}[k], \\
& \left.\tilde{h}_{1,2 K}[k] \tilde{X}_{1}[k]+\tilde{Z}_{2 K}[k] \mid \tilde{h}_{14} \tilde{X}_{1}[k]+\tilde{Z}_{4}[k]\right) \\
& \left.-H\left(\tilde{Z}_{(2, \ldots, 2 K) \backslash 4}[k]\right)\right] \\
& \leq n(\log P+o(\log P))+n o(\log P) \text {, }
\end{aligned}
$$

where the last step follows as it may be shown that the Gaussian distribution maximizes conditional entropy, as done in [19, Equation (30), (31)], similar to [48, Lemma 1], and similar to (7), (8). Note also that the conditional entropy term involves a single-input, multiple output term, and hence is again bounded by $n o(\log P)$, due to the conditioning.

Similarly, in the opposite direction, let $M_{B}$ denote all the messages except $M_{21}, M_{43}$ :

$$
\begin{aligned}
& n\left(R_{21}+R_{43}-\epsilon\right) \\
& \leq I\left(M_{21} ; \tilde{Y}_{1}^{n} \mid M_{B}\right)+I\left(M_{43} ; \tilde{Y}_{1}^{n}, \tilde{Y}_{(2, \ldots, 2 K) \backslash 4}^{n} \mid M_{21}, M_{B}\right) \\
& \leq H\left(\tilde{Y}_{1}^{n} \mid M_{B}\right)-H\left(\tilde{Y}_{1}^{n} \mid M_{21}, M_{B}\right) \\
&+H\left(\tilde{Y}_{1}^{n}, \tilde{Y}_{(2, \ldots, 2 K) \backslash 4}^{n} \mid M_{21}, M_{B}\right) \\
&-H\left(\tilde{Y}_{1}^{n}, \tilde{Y}_{(2, \ldots, 2 K) \backslash 4}^{n} \mid M_{21}, M_{B}, M_{43}\right) \\
&= H\left(\tilde{Y}_{1}^{n} \mid M_{B}\right)-H\left(\tilde{Z}_{1}^{n}\right)+H\left(\tilde{Y}_{(2, \ldots, 2 K) \backslash 4}^{n} \mid M_{21}, M_{B}, \tilde{Y}_{1}^{n}\right) \\
&-H\left(\tilde{Z}_{(2, \ldots, 2 K) \backslash 4}^{n}\right) \\
& \leq \sum_{k=1}^{n}\left[H\left(\tilde{Y}_{1}[k]\right)-H\left(\tilde{Z}_{1}[k]\right)\right. \\
&+ H\left(\tilde{Y}_{(2, \ldots, 2 K) \backslash 4}[k] \mid \tilde{Y}_{(2, \ldots, 2 K) \backslash 4}^{k-1}, M_{21}, M_{B}, \tilde{Y}_{1}^{n}, \tilde{X}_{1}^{n}, \tilde{X}_{(2, \ldots, 2 K)}^{k}\right. \\
&\left.\quad-H\left(\tilde{Z}_{(2, \ldots, 2 K) \backslash 4}[k]\right)\right] \\
& \leq n(\log P+o(\log P)) \\
&+ \sum_{k=1}^{n}\left[H \left(\tilde{h}_{42}[k] \tilde{X}_{4}[k]+\tilde{Z}_{2}[k], \tilde{h}_{43}[k] \tilde{X}_{4}[k]+\tilde{Z}_{3}[k],\right.\right. \\
& \quad-1
\end{aligned}
$$

$$
\begin{aligned}
& \tilde{h}_{45}[k] \tilde{X}_{4}[k]+\tilde{Z}_{5}[k], \cdots \tilde{\mathbf{h}}_{4,2 K-1}[k] \tilde{X}_{4}[k]+\tilde{\mathbf{Z}}_{2 K-1}[k] \\
& \left.\tilde{h}_{4,2 K}[k] \tilde{X}_{4}[k]+\tilde{Z}_{2 K}[k] \mid \tilde{h}_{41} \tilde{X}_{4}[k]+\tilde{Z}_{1}[k]\right) \\
& \left.\quad-H\left(\tilde{Z}_{(2, \ldots, 2 K) \backslash 4}[k]\right)\right] \\
& \quad \leq n(\log P+o(\log P))+n o(\log P) .
\end{aligned}
$$

Then,

$$
\begin{aligned}
d_{12}+d_{34}+d_{21}+d_{43} & \leq \limsup _{P \rightarrow \infty} \frac{R_{12}+R_{34}+R_{21}+R_{43}}{\log (P)} \\
& \leq 1+0+0+1+0+0=2,
\end{aligned}
$$

Summing over all rate pairs (see Remark 7) leads to the theorem, showing that the causal MIMO relay cannot increase the DoF.

Remark 7. We are able to sum over all rate pairs because the asymmetry of the transformed network (multiple antennas at user $2 K-1$ only) does not affect the DoF. Intuitively this $\backslash$ is) because for a SIMO or MISO point-to-point channel, the DoF is still 1. More rigorously, consider the sum rate pair $R_{12}+R_{2 K-1,2 K}$ and using similar notation (now $M_{A}$ denotes all messages except $M_{12}, M_{2 K-1,2 K}$ ), and following the same steps as in bounding $R_{12}+R_{34}$, we notice that the bounds do not depend on the asymmetry and again lead to 1 DoF per pair: ${ }^{8}$

$$
\begin{aligned}
& n\left(R_{12}+R_{2 K-1,2 K}-\epsilon\right) \\
& \leq I\left(M_{2 K-1,2 K} ; \tilde{Y}_{2 K}^{n} \mid M_{A}\right) \\
&+I\left(M_{12} ; \tilde{Y}_{2 K}^{n}, \tilde{Y}_{(2, \ldots, 2 K-1)}^{n} \mid M_{2 K-1,2 K}, M_{A}\right) \\
& \leq \ldots \\
& \leq \sum_{k=1}^{n}\left[H\left(\tilde{Y}_{2 K}[k]\right)-H\left(\tilde{Z}_{2 K}[k]\right)\right. \\
&+ \sum_{k=1}^{n}\left[H \left(\tilde{h}_{12}[k] \tilde{X}_{1}[k]+\tilde{Z}_{2}[k], \tilde{h}_{13}[k] \tilde{X}_{1}[k]+\tilde{Z}_{3}[k], \tilde{h}_{14}[k] \tilde{X}_{1}[k]+\tilde{Z}_{4}[k]\right.\right. \\
&\left.\tilde{\mathbf{h}}_{1,2 K-1}[k] \tilde{X}_{1}[k]+\tilde{\mathbf{Z}}_{2 K-1}[k] \mid \tilde{h}_{1,2 K} \tilde{X}_{1}[k]+\tilde{Z}_{2 K}[k]\right) \\
&\left.\quad-H\left(\tilde{Z}_{(2, \ldots, 2 K-1)}[k]\right)\right] \\
& \leq n(\log P+o(\log P))+n o(\log P)
\end{aligned}
$$

Thus we will have $d_{12}+d_{2 K-1,2 K} \leq 1$. Similar arguments follow for the opposite direction.

Note that we can reduce the number of antennas at the causal relay by one while still achieving $K$ DoF in two time slots: in slot 1 all users transmit their message and the $(2 K-1)$-antenna relay decodes $K$ linear combinations of 2 messages, i.e., $s_{12}$ and $s_{21}, s_{34}$ and $s_{43}, \ldots s_{2 K-1,2 K}$ and $s_{2 K, 2 K-1}$ (note to do this at least $2 K-1$ antennas are required). In slot 2 , the relay broadcasts the weighted sum of the decoded signals. By carefully choosing the $(2 K-1)$ dimensional beamforming vectors, $2 K-2$ interference signals may be eliminated at each receiver and it is able to decode the linear combination of its desired signal and the SI signal. By subtracting off the SI, each user may decode 1 desired signal in 2 time slots, i.e. in total $K$ DoF are achieved. Again note

\footnotetext{
${ }^{8}$ We leave out several steps and replace it with $\cdots$ to avoid repetition, as these follow identically.
} 
that this scheme does not require infinite time or frequency symbol extensions and thus can reduce the complexity of the transceivers.

Remark 8. We finally remark on the relationship between this work and some on multi-way relay channels. The general results of [28] on the DoF of MIMO multi-way relay channels is able to capture our scenario partially: in particular, while the model in [28] may capture $K$ pairs of users wishing to exchange messages with the help of a MIMO relay, as in our case, it does not consider direct links between all users in the channel - i.e. all users are connected through the relay only. Applying [28, Theorem 4] to our scenario, we may conclude that if there are no direct links between users, then

$D o F=2 K$ if the number of antennas at relay is at least $2 K$, which is the same as our result for instantaneous relays, except that we have direct links between all users as well, and they have causal relays instead of instantaneous ones. The same result was shown in [25], i.e. that $2 K$ DoF may be achieved with by a causal, half-duplex relay with at least $2 K$ antennas when there are no direct links between the users. Thus, in addition to prior conclusions concerning instantaneous versus causal relays, we are also able to conclude that the presence of the direct links actually reduces the DoF compared to when there are no direct links, for causal relays.

\section{CONCLUSION}

We proposed and studied bi-directional interference networks with and without a MIMO relay where all nodes are connected and operate in full duplex. We demonstrated that the degrees of freedom of the $K$-pair-user full-duplex bidirectional interference network without a relay is $K$. This indicates that full-duplex operation doubles the DoF over the setting with time-sharing half-duplex nodes, but that interaction, or adapting transmission based on previously received signals at the users, cannot further increase the DoF beyond what full-duplex allows, i.e. the DoF is just that of two one-way, non-interactive ICs. We next showed that if we introduce a $2 K$ antenna, full-duplex and non-causal relay, that the DoF may again be doubled over the full-duplex, relay-free counterpart (or quadrupled over the time-sharing half-duplex counterpart). We demonstrated a one-shot scheme to achieve the maximal $2 K$ DoF. In sharp contrast, if the relay is causal rather than non-causal, we derived a new converse showing that the DoF cannot be increased beyond $K$ for a $K$-pair-user full-duplex bi-directional interference network. We commented on how one may decrease the number of antennas at the relay node, at the expense of either a reduced achievable DoF or cognition at the relay. Another possible avenue to investigate whether one can reduce the number of antennas needed at the relay may be to use lattice coding and network coding. However, a converse for the $K$-pair user full-duplex bi-directional interference networks with an instantaneous relay with fewer than $2 K$ antennas is open. Overall, this work has shown that in $K$ pair-user full-duplex bi-directional interference networks, fullduplex operation at least doubles the achievable DoF (over time-sharing half-duplex systems), interaction does not help (unless some channel gains are zero), and a full-duplex relay may further increase the DoF (quadrupling the DoF over a half-duplex system) if it is instantaneous and has a sufficient number of antennas.

\section{REFERENCES}

[1] Z. Cheng and N. Devroye, "The degrees of freedom of the K-pair-user full-duplex two-way interference channel with a MIMO relay," in Proc. IEEE Int. Symp. Inf. Theory, Honolulu, Jul. 2014.

[2] _ , "Degrees of freedom of the two-way interference channel with a non causal multi-antenna relay," in Proc. IEEE Global Telecommun. Conf., Atlanta, Dec. 2013.

[3] K. Tsubouchi, H. Nakase, A. Namba, and K. Masu, "Full duplex transmission operation of a $2.45-\mathrm{GHz}$ asynchronous spread spectrum using a san convolver," Ultrasonics, Ferroelectrics and Frequency Control, IEEE Transactions on, vol. 40, no. 5, pp. 478-482, Sept 1993.

[4] S. Chen, M. Beach, and J. McGeehan, "Division-free duplex for wireless applications," Electronics Letters, vol. 34, no. 2, pp. 147-148, Jan 1998.

[5] A. Khandani, "Methods for spatial multiplexing of wireless two-way channels," Oct. 19 2010, US Patent 7,817,641. [Online]. Available: http://www.google.com/patents/US7817641

[6] B. Radunovic, D. Gunawardena, P. Key, A. Proutiere, N. Singh, V. Balan, and G. DeJean, "Rethinking indoor wireless mesh design: Low power, low frequency, full-duplex," in Wireless Mesh Networks (WIMESH 2010), 2010 Fifth IEEE Workshop on, June 2010, pp. 1-6.

[7] M. Jain, J. Choi, T. Kim, D. Bharadia, S. Seth, K. Srinivasan, P. Levis, S. Katti, and P. Sinha, "Practical, real-time, full duplex wireless," in Mobicom, Las Vegas, NV, Sep. 2011.

[8] A. Sahai, G. Patel, and A. Sabharwal, "Asynchronous full-duplex wireless," in International Conference on Communication Systems and Networks (COMSNETS), Jan. 2012.

[9] T. Han, "A general coding scheme for the two-way channel," IEEE Trans. Inf. Theory, vol. IT-30, pp. 35-44, Jan. 1984.

[10] Z. Cheng and N. Devroye, "On the capacity of multi-user two-way linear deterministic channels," in Proc. IEEE Int. Symp. Inf. Theory, Cambridge, Jul. 2012.

[11] _ " "Two-way networks: when adaptation is useless," IEEE Trans. Inf. Theory, vol. 60, no. 3, pp. 1793-1813, Mar. 2014.

[12] _ " "On constant gaps for the two-way gaussian interference channel," in Proc. Allerton Conf. Commun., Control and Comp., Oct. 2012.

[13] C. Suh, I.-H. Wang, and D. Tse, "Two-way interference channels," in Proc. IEEE Int. Symp. Inf. Theory, Cambridge, Jul. 2012.

[14] P. Rost, "Robust and efficient multi-cell cooperation under imperfect CSI and limited backhaul," Wireless Communications, IEEE Transactions on, vol. 12, no. 4, pp. 1910-1922, April 2013.

[15] R. Etkin, D. Tse, and H. Wang, "Gaussian interference channel capacity to within one bit," IEEE Trans. Inf. Theory, vol. 54, no. 12, pp. 55345562, Dec. 2008.

[16] Z. Cheng and N. Devroye, "On constant gaps for the K-pair user twoway Gaussian interference channel with interaction," in Proc. of IEEE Global Conf. on Signal and Information Processing (GlobalSIP), Austin, Dec. 2013.

[17] L. Zheng and D. Tse, "Communication on the grassmann manifold: a geometric approach to the noncoherent multiple-antenna channel," IEEE Trans. Inf. Theory, vol. 48, no. 2, pp. 359-383, 2002.

[18] V. Cadambe and S. Jafar, "Interference alignment and the degrees of freedom for the K-user interference channel," IEEE Trans. Inf. Theory, vol. 54, no. 8, pp. 3425-3441, Aug. 2008.

[19] —, "Degrees of freedom of wireless networks with relays, feedback, cooperation and full duplex operation," IEEE Trans. Inf. Theory, vol. 55, no. 5, pp. 2334-2344, May 2009.

[20] S. A. Jafar and S. Shamai, "Degrees of freedom region of the MIMO X channel," IEEE Trans. Inf. Theory, vol. 54, no. 1, pp. 151-170, Jan. 2008.

[21] R. Etkin and E. Ordentlich, "The degrees-of-freedom of the K-user Gaussian interference channel is discontinuous at rational channel coefficients," IEEE Trans. Inf. Theory, vol. 55, no. 11, pp. 4932-4946, Nov. 2009.

[22] L. Ke, A. Ramamoorthy, Z. Wang, and H. Yin, "Degrees of freedom region for an interference network with general message demands," in Proc. IEEE Int. Symp. Inf. Theory, 2011, pp. 36-40.

[23] C. Vaze and M. Varanasi, "The degrees of freedom region of the twouser mimo broadcast channel with delayed CSIT," in Proc. IEEE Int Symp. Inf. Theory, 2011, pp. 199-203. 
[24] N. Lee and R. Heath, "Multi-way information exchange over completely-connected interference networks with a multi-antenna relay," 2013. [Online]. Available: http://arxiv.org/abs/1302.0749

[25] H. Ghozlan, Y. Mohasseb, H. El Gamal, and G. Kramer, "The MIMO wireless switch: Relaying can increase the multiplexing gain," in Information Theory, 2009. ISIT 2009. IEEE International Symposium on, June 2009, pp. 1448-1452.

[26] C. Wang and S. Jafar, "Degrees of freedom of the two-way relay MIMO interference channel," 2013. [Online]. Available: http://escholarship.org/uc/item/9qc3343h

[27] N. Lee and C. Wang, "Aligned interference neutralization and the degrees of freedom of the two-user wireless networks with an instantaneous relay," IEEE Trans on Comm., vol. PP, no. 99, pp. 1-9, 2013.

[28] Y. Tian and A. Yener, "Degrees of freedom for the MIMO multi-way relay channel," IEEE Trans. Inf. Theory, vol. 60, no. 5, pp. 2495-2511, May 2014

[29] A. G. D. Gunduz, A. Yener and H. Poor, "The multiway relay channel," IEEE Trans. Inf. Theory, vol. 59, no. 1, pp. 51-63, Jan. 2013.

[30] A. Chaaban, A. Sezgin, and A. Avestimehr, "Approximate sum-capacity of the Y-channel," IEEE Trans. Inf. Theory, vol. 59, no. 9, pp. 57235740, Sep. 2013.

[31] N. Lee, J.-B. Lim, and J. Chun, "Degrees of freedom of the MIMO Y channel: Signal space alignment for network coding," IEEE Trans. Inf. Theory, vol. 56, no. 7, pp. 3332-3342, July 2010.

[32] H. Mu and J. Tugnait, "Achievable degrees of freedom for $\mathrm{K}$-user MIMO Y channels using signal group based alignment," IEEE Trans. Wireless Comm., vol. 13, no. 8, pp. 4520-4533, Aug 2014.

[33] K. Liu and M. Tao, "Generalized signal alignment: On the achievable dof for multi-user MIMO two-way relay channels," CoRR, vol. abs/1405.0718, 2014. [Online]. Available: http://arxiv.org/abs/1405.0718

[34] M. Maddah-Ali, A. Motahari, and A. Khandani, "Communication over $\mathrm{X}$ channel: Signaling and performance analysis," in Univ. of Waterloo, Waterloo, ON, Canada, Tech. Rep. UW-ECE-2006-27, Dec. 2006.

[35] S. Jafar, "Interference alignment: A new look at signal dimensions in a communication network," in Foundations and Trends in Communications and Information Theory, no. 1, 2011, pp. 1-134.

[36] A. Motahari, S. Oveis-Gharan, M. Maddah-Ali, and A. Khandani, "Real interference alignment: Exploiting the potential of single antenna systems," vol. 60, no. 8, pp. 4799-4810, Aug. 2014.

[37] S. Jafar and S. Vishwanath, "Generalized degrees of freedom of the symmetric Gaussian user interference channel," IEEE Trans. Inf. Theory, vol. 56, no. 7, pp. 3297-3303, Jul. 2010.

[38] S. Mohajer, R. Tandon, and H. Poor, "Generalized degrees of freedom of the symmetric K-user interference channel with feedback," in Proc. IEEE Int. Symp. Inf. Theory, 2012, pp. 3125-3129.

[39] I. Shomorony and S. Avestimehr, "Degrees of freedom of two-hop wireless networks: Everyone gets the entire cake," in Proc. Allerton Conf. Commun., Control and Comp., Oct. 2012.

[40] A. El Gamal and N. Hassanpour, "Relay-without-delay," in Proc. IEEE Int. Symp. Inf. Theory, 2005, pp. 1078-1080.

[41] G. Kramer, "Outer bounds on the capacity of Gaussian interference channels," IEEE Trans. Inf. Theory, vol. 50, no. 3, Mar. 2004.

[42] D. Tuninetti, "An outer bound region for Interference Channels with Generalized Feedback," in Information Theory and Applications Workshop (ITA), 2010, 2010.

[43] — , "K-user interference channels: General outer bound and sumcapacity for certain Gaussian channels," in Proc. IEEE Int. Symp. Inf. Theory, St. Petersburg, Aug. 2011.

[44] A. Goldsmith, Wireless Communications, 2nd ed. Cambridge University Press, 2005.

[45] S. Krishnamurthy, A. Ramakrishnan, and S. Jafar, "Degrees of freedom of rank-deficient MIMO interference channels," IEEE Trans. Inf. Theory, vol. 61, no. 1, pp. 341-365, Jan 2015.

[46] T. Liu, D. Tuninetti, and S.-Y. Chung, "On the DoF region of the two-user interference channel with an instantaneous relay," http://arxiv.org/abs/1506.06558.

[47] N. Devroye, P. Mitran, and V. Tarokh, "Achievable rates in cognitive radio channels," IEEE Trans. Inf. Theory, vol. 52, no. 5, pp. 1813-1827, May 2006.

[48] A. Host-Madsen, "Capacity bounds for cooperative diversity," IEEE Trans. Inf. Theory, vol. 52, pp. 1522-1544, Apr. 2006.

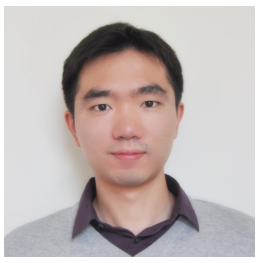

Zhiyu Cheng Zhiyu Cheng received the B.S. degree in information engineering from the East China University of Science and Technology, Shanghai, China, in 2009, and the M.S. and the Ph.D. degrees in electrical and computer engineering from the University of Illinois at Chicago (UIC), Chicago, IL, in 2012 and 2014 respectively.Dr. Cheng'sresearch interests include multi-user information theory and wireless full-duplex two-way communication networks.He was a summer research intern at Bell Laboratories, Alcatel-Lucent, Murray Hill, NJ, in 2013.

He was a recipient of the China Scholarship Council Graduate Fellowship for the years 2009-2013.Currently Dr. Cheng is a systems \& architecture engineer III at NXP Semiconductors in Austin, TX.

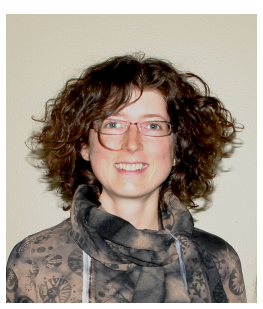

Natasha Devroye Natasha Devroye is an Associate Professor in the Department of Electrical and Computer Engineering at the University of Illinois at Chicago (UIC), which she joined in January 2009. From July 2007 until July 2008 she was a Lecturer at Harvard University. Dr. Devroye obtained her $\mathrm{Ph} . \mathrm{D}$ in Engineering Sciences from the School of Engineering and Applied Sciences at Harvard University in 2007, and a Honors B. Eng in Electrical Engineering from McGill University in 2001. Dr. Devroye was a recipient of an NSF CAREER award in 2011 and was named UIC's Researcher of the Year in the "Rising Star" category in 2012. She has been an Associate Editor for IEEE Transactions on Wireless Communications, IEEE Journal of Selected Areas in Communications, and is currently an Associate Editor for the IEEE Transactions on Cognitive Communications and Networking. Her research focuses on multiuser information theory and applications to cognitive and software-defined radio, radar, relay and two-way communication networks.

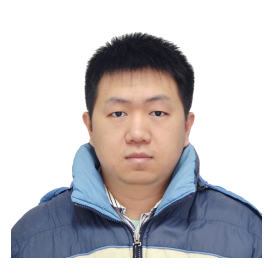

Tang Liu Tang Liu received his B.S. degree in telecommunication engineering from University of Electronic Science and Technology of China (UESTC), Chengdu, China, in 2010, and M.S. degree in electrical engineering from Korea Advanced Institute of Science and Technology (KAIST), Daejeon, South Korea, in 2013. He is currently a Ph.D. student in University of Illinois at Chicago (UIC), Chicago. His research interests are in interference channel, network coding, and index coding. He was 2010 to 2013 awarded the Korean Government Scholarship from 